\title{
THE RELATIONSHIP BETWEEN MALE STERILITY AND MEMBRANE LIPID PEROXIDATION AND ANTIOXIDANT ENZYMES IN WHEAT (Triticum aestivum L.)
}

\author{
Hongzhan LIU ${ }^{1,2}$, Gaisheng ZHANG ${ }^{1, *}$, Junsheng WANG $^{2}$, Qingsong BA ${ }^{1}$, Huixue CHE ${ }^{1}$, Yulong \\ SONG ${ }^{l}$, Pengfei ZHANG ${ }^{l}$, Na NIU ${ }^{l}$, Junwei WANG ${ }^{1}$, Shoucai MA ${ }^{l}$, Long CHEN ${ }^{2}$ \\ ${ }^{I}$ Northwest A\&F University, National Yangling Agricultural Biotechnology \& Breeding Center, Yangling \\ Branch of State Wheat Improvement Centre, Wheat Breeding Engineering Research Center, Ministry of \\ Education, Key Laboratory of Crop Heterosis of Shaanxi Province, Yangling, Shaanxi, P.R. CHINA \\ ${ }^{2}$ College of Life Science and Agronomy, Zhoukou Normal University, Zhoukou, \\ Henan, P.R. CHINA \\ *Corresponding author: zhanggaisheng18@sohu.com
}

Received: 12.10 .2015

\begin{abstract}
Malondialdehyde (MDA), which is thought to be important inducible factor of cell apoptosis if excessively accumulated in cells, is often regarded as a widely used marker of oxidative lipid injury whose concentration varies in response to biotic and abiotic stress. To elucidate the metabolic mechanism of ROS production and scavenging of the male sterility, normal fertility and male sterility wheat, including genetic and physiologically male sterility, by comparing MDA content and antioxidant enzymes changes in the sterile and fertile leaves, spikes, florets, rachillaes, ovaries and anthers at different developmental anther stages, were employed for studying the relationship between the male sterility and membrane lipid peroxidation, antioxidant enzymes. The results showed that tissue of male sterility had roughly higher content of MDA than corresponding fertile plants, and ovary performance was not obvious but most obvious in anther. Simultaneously, the higher activities of antioxidant enzymes were measured in the anthers of male sterility plant, in which an increase of ROS in anthers of abortion stage had an inducible effect on the antioxidant enzymes. MDA content and scavenging-enzyme activities had difference in the sterile and fertile leaves, spikes, florets, rachillaes at different anther stages, indicating that different tissues of ROS metabolism had noticeable difference in male sterility and normal wheat. It can be considered that the disorder of scavenging-enzyme activities and accumulation of MDA in the male organ, and supplier of nutrition blades were causing a further substance metabolism disorder, energy loss, leading to the occurrence of male sterility.
\end{abstract}

Key words: Wheat - male sterility - malondialdehyde - antioxidant enzymes

Abbreviations: CAT---Catalase; CMS---Cytoplasmic male sterility; CHA---Chemical Hybridization Agent; GS---Cytoplasmic male sterile line (S)-1376; MDA---Malondialdehyde; NW---Wheat cultivar 1376; PMS--CHA-SQ-1 induced male sterility wheat; POD---Peroxidase; ROS---Reactive oxygen species; SOD--Superoxide dismutase; SEM---Scanning electronm icroscopy.

\section{INTRODUCTION}

In the process of plant growth and development, antioxidant enzymes play an important role not only in the biochemical signs but also in male sterility (Deng et al., 2012; Jiang et al., 2007). Reactive oxygen species (ROS) is a by-product in the process of aerobic metabolism. In vivo, if ROS are at high level sustaining, the organism will suffer oxidative stress, resulting in protein and nucleic acid damage, lipid peroxidation and even necrocytosis (Deng et al., 2012; Fridovich, 1978). Malondialdehyde is often regarded as a widely used marker of oxidative lipid injury whose concentration varies in response to biotic and abiotic stress (Sunkar et al., 2003). As an important scavenging mechanism, antioxidant enzymes display a critical role in the plant development (Bailly, 2004). And several enzymes are involved in the detoxification of ROS, such as Superoxide dismutase (SOD), peroxidase (POD) and catalase (CAT) (Bhaskaran and Panneerselvam, 2013; Ou et al., 2013).

A considerable amount of literatures had reported the content of MDA had been associated with male sterility (Draganic et al. 2011; Li et al., 2004; Liu et al., 2012; Mi et al., 2008; Fatma et al., 2010). And MDA as final product of lipid peroxidation (LPO) could be reflected the degree of cell membrane damage according to its content high or low. MDA can alter proteins, DNA, RNA, and other 
biomolecules through Schiff's base addition reactions. LPO, in both organelle and cellular membranes, takes place when the ROS levels are reached the normal threshold. The occurrence of LPO is not only directly affecting normal cellular functioning, but also aggravating the oxidative stress through production of lipid-derived radicals (Gill and Tuteja, 2010). Jiang and Zhang (Jiang and Zhang, 2001) reported that treatment with lower concentrations of $\mathrm{ABA}$ led to a relatively increase in the levels of MDA and induced the antioxidant defence systems against oxidative stress. Darko' et al (Darkó et al., 2004) also believed that ROS metabolism condition changed when the plant tissues were subjected to the stress. In rice, the researcher found that excessive ROS were accumulated in the cytoplasmic male sterility line anthers. In wheat, metabolic mechanism of ROS was significant impact in the pollen abortion (Ba et al., 2013; Wang et al., 2009).

Superoxide dismutase (SOD), as ubiquitous in plantage, is the most effective intracellular enzymatic antioxidant and the first line of defense against the toxic effects of elevated levels of ROS (Mittler, 2002). The study of the Mn-SOD activity in different development stages among different male sterile lines in wheat showed that SOD had a high relationship of male sterility. Peroxidases (POD) are involved in a broad range of metabolism processes throughout the plant life cycle, including the metabolism of ROS, auxin metabolism, lignin and suberin formation, cross-linking of cell wall components and phytoalexin synthesis. In maize, POD activity in the male sterility line was higher than its maintainer and restorer line in every stages of florets development (Jia et al., 2009). Catalases (CAT) play a key role in the removal of $\mathrm{H}_{2} \mathrm{O}_{2}$ generated in peroxisomes by oxidases and its isozymes have been studied extensively in higher plants (Polidoros and Scandalios, 1999). The expression of fertility is the results as physiological process controlled by gene, a biochemical reaction and form building in plants, including a series of substance metabolism and energy metabolism, involving a variety of complex physiological and biochemical.

In wheat hybrid breeding, CMS and CHA induced wheat male sterility was the most effective way. In the present study, our data showed that tissue of male sterility had roughly higher content of MDA than corresponding fertile plants, and ovary performance was not obvious but most obvious in anther.

\section{MATERIALS AND METHODS}

Plant growth and materials collection

Cytoplasmic male sterile line (S)-1376 and wheat cultivar 1376 were grown in the experimental field of Northwest A\&F University, located in Yangling, China, on October 8, 2012. In the next April, when wheat development to the period of female stamens primordia formation to connectivum, wheat cultivar 1376 were sprayed with $5 \mathrm{~kg} \mathrm{hm}-2$ dose CHA-SQ-1, and the control was spraying with water. GS and its maintainer line 1376 and PMS wheat with the same nuclear background were used in this study. All the materials were collected according to the development of anther of 1376. These operations were carried out at $0-4{ }^{\circ} \mathrm{C}$. Plant samples were frozen in liquid nitrogen and stored at $-80^{\circ} \mathrm{C}$ until further analysis. We collected the leaves, the spikes, the florets, the rachillaes, the ovaries and the anthers as the development stage of anther, which is the stage of meiophase, tetrad, mononuclear, binuclear and trinuclear.

\section{Histological analysis and phenotypic characterization}

Nuclei of wheat anthers at various developmental stages was staining with carbol fuchsin. SEM were performed as described by Dou et al (Dou et al., 2011). Pollen grains of the trinuclear stage anthers were stained with $1 \% \mathrm{KI}_{2} \mathrm{I}_{2}$.

\section{Assays of enzyme activities and MDA content}

In brief, frozen samples $(0.5 \mathrm{~g})$ were finely powdered in liquid nitrogen with a pestle in a pre-cooled mortar and homogenized with chilled $50 \mathrm{~m} \mathrm{~mol} \mathrm{~L}^{-1}$ phosphate $(\mathrm{pH} 7.0)$ with three repeats. The homogenate was centrifuged at $4{ }^{\circ} \mathrm{C}$ for $20 \mathrm{~min}$ at $10000 \times \mathrm{g}$ and the resulting supernatants were used to measure the activities of enzyme activities and the content of MDA by a spectrophotometer (Evolution 300/600, Thermo Scientific, America).

SOD activities were measured according to the inhibition of the photochemical reduction of nitro-blue tetrazolium (NBT) (Winterbourn et al., 1975). POD activity was determined according to the method of the oxidation of guaiacol, using hydrogen peroxide. Briefly, $0.05 \mathrm{~mL}$ of the enzyme extract were reacted with $4 \mathrm{~mL}$ of $0.3 \%(\mathrm{w} / \mathrm{v})$ guaiacol compounded with $20 \mathrm{~m} \mathrm{~mol} \mathrm{~L}^{-1}$ phosphate (pH 6.0) and $0.05 \mathrm{ml}$ of $1 \% \mathrm{H}_{2} \mathrm{O}_{2}(\mathrm{v} / \mathrm{v})$. Adequate mixing the reaction mixture, and then analyzed for absorbance at 470 nm. CAT activities were determined spectrophotometrically by monitoring the decrease in absorbance at $240 \mathrm{~nm}$. CAT activity was tested following the $\mathrm{H}_{2} \mathrm{O}_{2}$ decomposition. One unit of the enzyme was defined as an optical density value change of 0.01 . The content of MDA was determined using the modified procedure as described by Hodges et al (Hodges et al., 1999). The absorbance was recorded at 450, 532 and 600 $\mathrm{nm}$. The solution concentration of MDA $\left(\mu \mathrm{mol} \mathrm{L} \mathrm{L}^{-1}\right)$ and the content of MDA ( $\mu \mathrm{mol} \mathrm{g}^{-1}$ ) were calculated with formula.

\section{RESULTS}

According to "Plant growth and materials collection", samples morphology was observed and cells were examined under a light microscope to determine the different developmental stage (Figs 1a to 1e) and then collected materials 

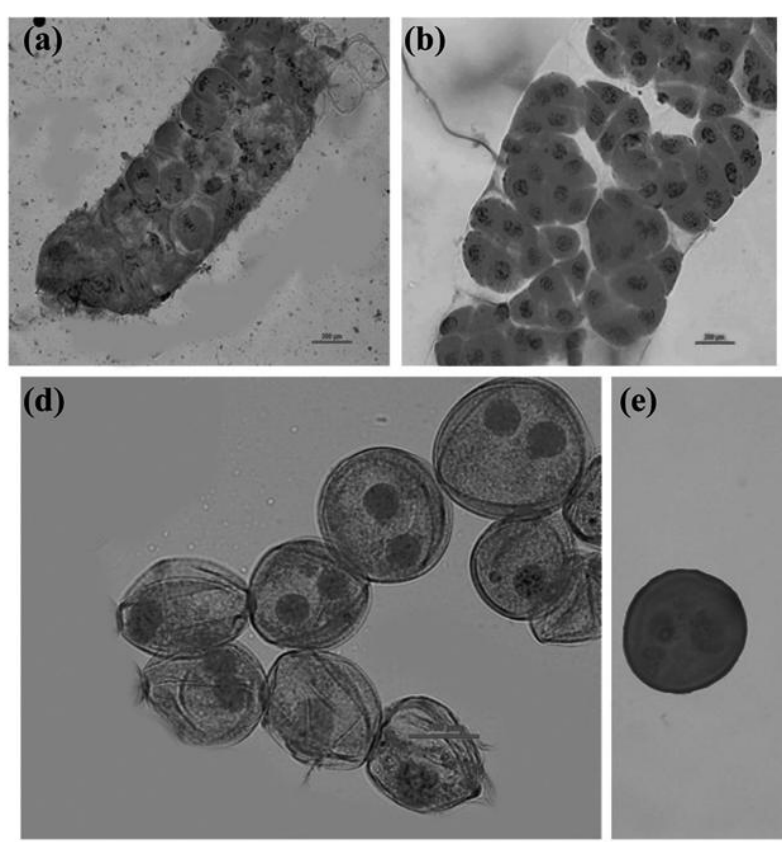

(e)

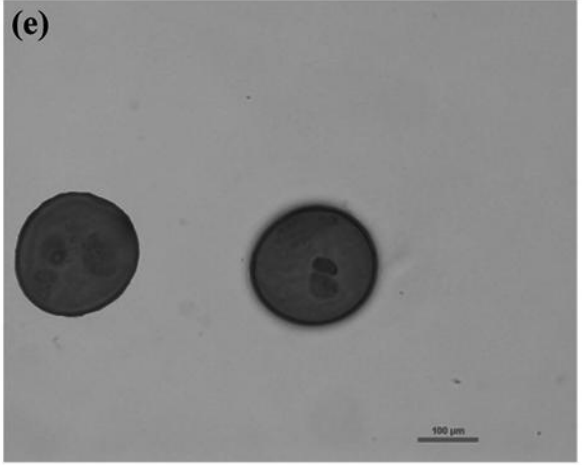

Fig 1. Each stage of microspore: meiophase stage (a), tetrad stage (b), mononuclear stage(c), binuclear stage(d) and trinuclear stage(e). Bars $=100 \mu \mathrm{m}$ in (a) to (e).

\section{Phenotypic analysis}

The effect of CHA SQ-1 on wheat male sterility was evaluated and the results were summarized in Table 1 . The relative male sterility rate in SQ-1-sprayed wheat was almost complete (up to $99.79 \%$ ) and seed-setting rate of artificial saturation pollination was high $(94.55 \%)$ without causing pistil damage. The relative male sterility rate of GS was $100 \%$, and seed-setting rate of artificial saturation pollination was $98 \%$. As shown in Fig 2a, the NW pollen grains which stained with $\mathrm{KI}^{-\mathrm{I}_{2}}$ should be full starch accumulation, however, the pollen grains of PMS and GS plants should be almost no starch accumulation (Figs 2b and 2c). For further analysis, the results of pollen grains of SEM showed that the NW pollen grains had a smooth and particulate exine patterning and nearly round shape (Figure 2d), whereas the pollen shape of PMS and GS plants appeared severely malformation (Figures $2 \mathrm{e}$ and $2 \mathrm{f}$ ). Compared with the well-formed exterior of NW anthers (Fig 2g), the PMS anther exterior was slightly disorderly and the anther of GS was before unsystematic (Figs $2 \mathrm{~h}$ and 2i). From these marked differences, the direct response object of male sterility is anther, but the result of male sterility could be the result of whole plant physiological and metabolic changes, especially to provide nutrition of leaves and to the anthers survival of spikes. So the research was not only focus on the change of MDA and antioxidant enzymes about the anthers of different male sterility line on the different stage, but also the leaves, the floret, the rachilla, the ovary and the spikes (Fig 3).

Table 1. Relative male sterility ratio with seed setting percentage of artificial saturation pollination induced by CHA SQ-1.

\begin{tabular}{|c|c|c|c|c|c|}
\hline \multirow[t]{2}{*}{ Material } & \multicolumn{3}{|c|}{$\begin{array}{l}\text { Averaging grain per panicle of different } \\
\text { treatments }\end{array}$} & \multirow[t]{2}{*}{$\operatorname{RMSR}(\%)^{\mathrm{d}}$} & \multirow[t]{2}{*}{$\operatorname{ASPSP}(\%)^{\mathrm{e}}$} \\
\hline & ANW $^{\mathbf{a}}$ & $\mathbf{A A P}^{\mathbf{b}}$ & $\mathbf{A A S P}^{\mathbf{c}}$ & & \\
\hline 1376 & 37.60 & 0.08 & 35.63 & 99.79 & 94.55 \\
\hline
\end{tabular}

${ }^{\mathrm{a} A v e r a g i n g ~ g r a i n s ~ p e r ~ p a n i c l e ~ o f ~ n o r m a l ~ w h e a t . ~}$

${ }^{\mathrm{b}}$ Averaging grains per panicle of PMS autocopulation pollination.

${ }^{\mathrm{c} A v e r a g i n g ~ g r a i n s ~ p e r ~ p a n i c l e ~ o f ~ P M S ~ a r t i f i c i a l ~ s a t u r a t i o n ~ p o l l i n a t i o n ~ s p i k e l e t s . ~}$

${ }^{\mathrm{d}}$ Relative male sterility rate.

${ }^{\mathrm{e}}$ Artificial saturation pollination setting percentage 

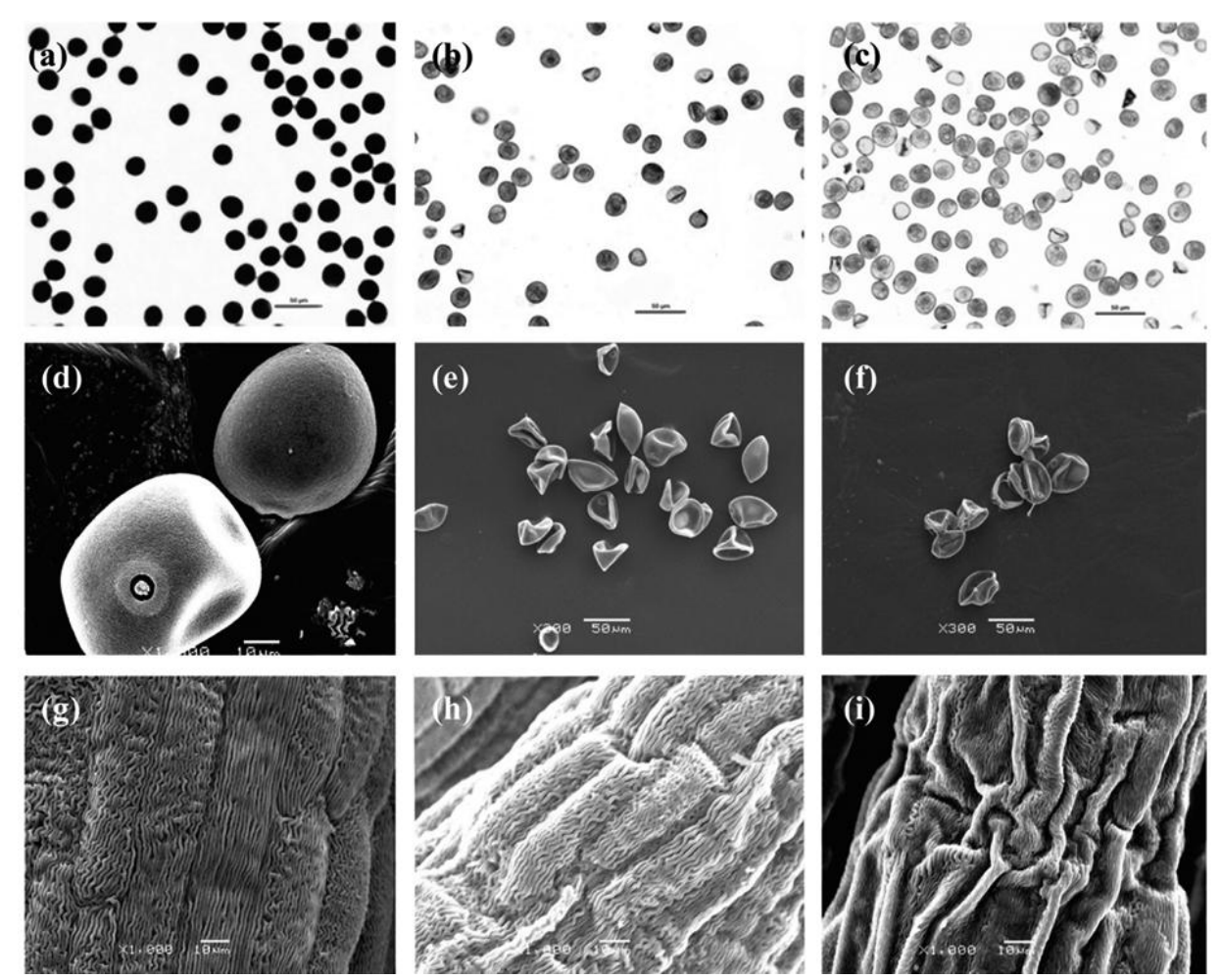

Fig 2. Morphological changes of the PMS and GS compared with complete fertility plants about anthers.

(a) to (c) Pollen grains stained with KI-I2 solution of fertility plants NW (a), PMS plants (b) and GS plants(c). (d) to (f) Scanning electron microscopy analysis of pollen grains of fertility plants NW (d), PMS plants (e) and GS plants(f). (g) to (i) Scanning electron microscopy analysis of the trinuclear stage anther surface of fertility plants NW (g), PMS plants (h) and GS plants(i).

Bars $=100 \mu \mathrm{m}$ in (a) to (c), $10 \mu \mathrm{m}$ in (d), $50 \mu \mathrm{m}$ in (e) and (f), $10 \mu \mathrm{m}$ in (g) to (i).

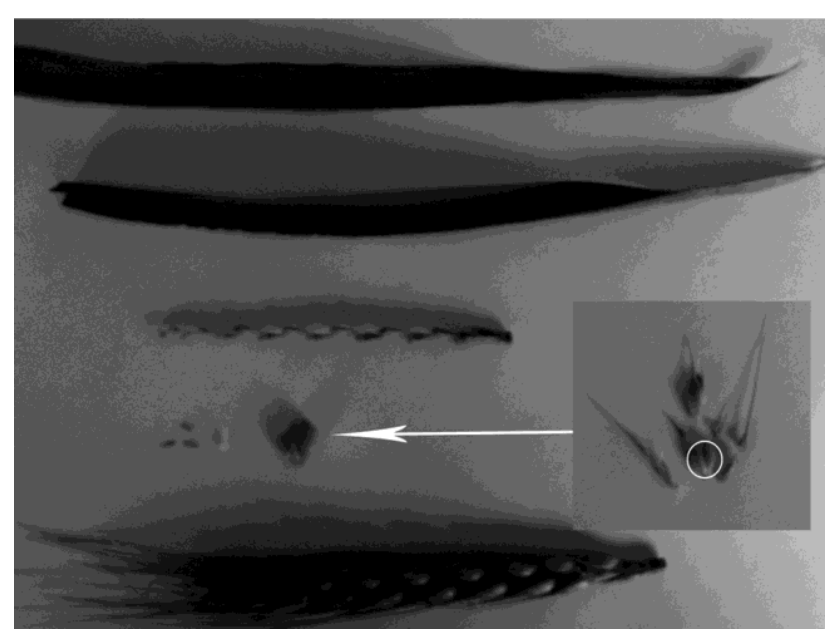

Fig 3. The collected materials. Bars $=10 \mathrm{~cm}$.

Determination of the activities of antioxidant enzymes and MDA content

In top second leaf, SOD activity is significantly lower than NW in the stage of meiophase in PMS and GS, but significantly higher in tetrad stage (Fig 4a). POD activity of top second leaf was significantly higher than normal wheat in PS. In the early mononuclear stage of GS, POD activity was lower than NW, but it was higher than NW in the other stages (Fig 4b). CAT activity was rising along with the stage of anther in the NW top second leaf. After tetrad stage, CAT activity was lower than NW in the top second leaf of PMS and GS, especially in the stage of early mononuclear stage (Fig 4c); then the content of MDA was higher than NW in the PMS and GS top second leaf, especially in the stage of early mononuclear stage (Fig 4d).

SOD of NW flag leaf was higher than that of PMS and GS in the stage of binuclear, and so were POD and CAT. For POD activity, there were significantly higher $(\mathrm{P}<0.01)$ than NW in the stage of meiophase and tetrad in PMS flag leaves. In GS flag leaves, POD activity had a trend of slowly rise but generally lower than that of NW. Then in the stage of trinuclear flag leaf, the PMS POD activity was significantly higher $(\mathrm{P}<0.05)$ than NW. CAT activity was significant lower $(\mathrm{P}<0.01)$ than $\mathrm{NW}$ in the stage of tetrad and binuclear in PMS and GS (Figs 4e, 4f and 4g). In the flag leaf, compared to the normal wheat, PMS had a significant increase $(\mathrm{P}<0.01)$ in the concentration of MDA in the anther stage of later mononuclear stage and binuclear stage, and GS had a significant increase $(\mathrm{P}<0.01)$ in the anther stage of binuclear stage (Fig 4h). 

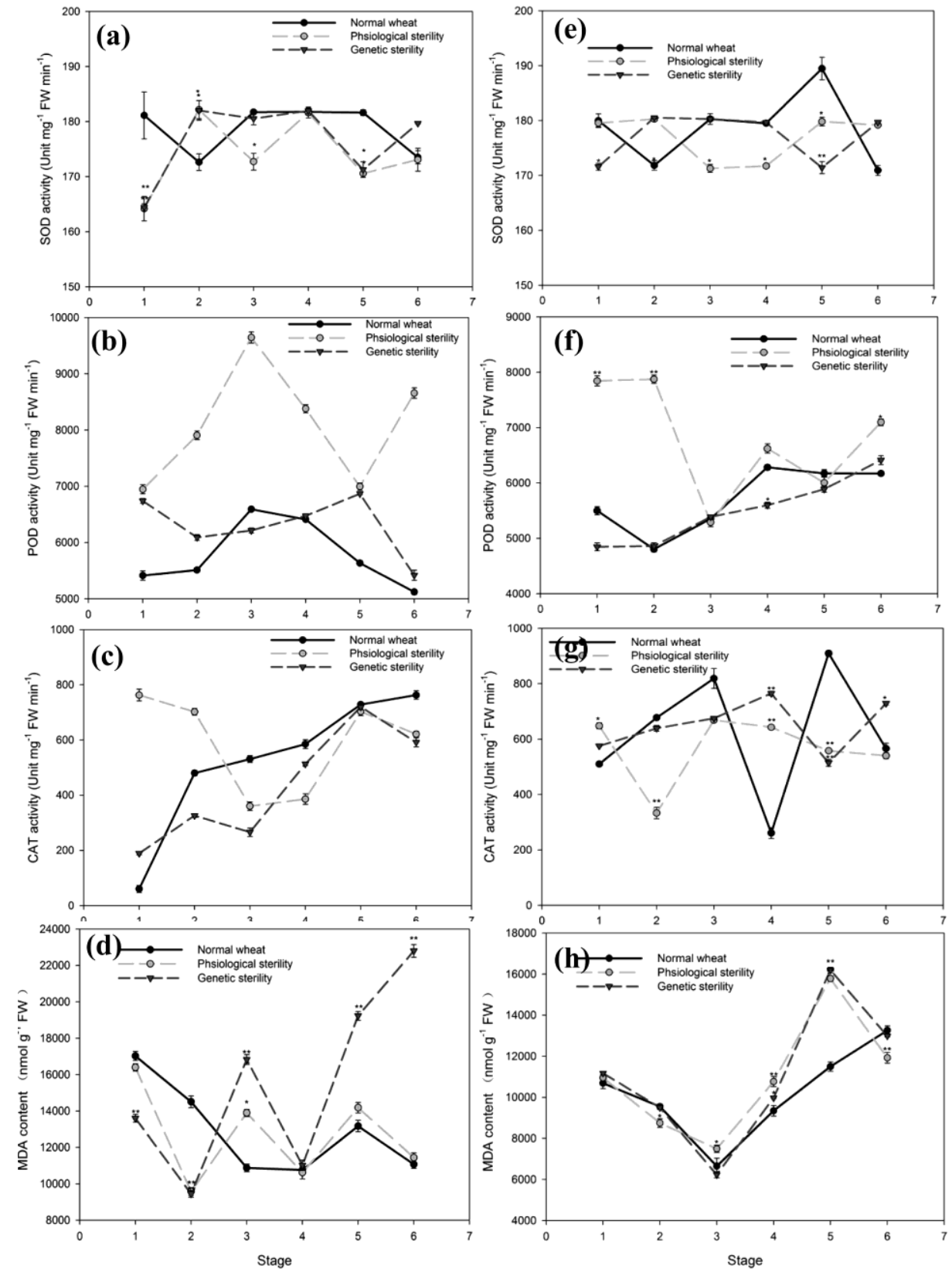

Fig 4. Activity of antioxidant enzymes and MDA content of top second leaf and flag leaf.

1, meiophase stage; 2, tetrad stage; 3 , early mononuclear stage; 4 , late mononuclear stage; 5 , binuclear stage; 6 , trinuclear stage. $n=3$, * Indicate $\mathrm{P}<0.05$ significant difference; $* *$ Indicate $\mathrm{P}<0.01$ significant difference. (collete materials according to anther development period).

In spikes, POD activity and CAT activity and MDA content had a similar trend along with the anther stages. And SOD activity was significantly higher $(\mathrm{P}<0.05)$ than NW in the PMS and GS. POD activity and CAT activity of GS spikes were lower than NW spikes in the whole process. POD activity of PMS spikes was higher than NW before mononuclear stage, while it was lower after mononuclear stage and reached significantly $(\mathrm{P}<0.01)$ in the stage of trinuclear. But CAT activity was significantly $(\mathrm{P}<0.01)$ higher than NW in the trinuclear stage (Figs 5a, 5b and 5c). MDA content of PMS and GS spikes were higher than that of NW in the period of tetrad stage to binuclear stage (Fig $5 d)$.
For SOD activity in the floret, NW present a "high-lowhigh" movement, but jumping up and down in the PMS and GS. In the stage of trinuclear, SOD was significant lower than NW in the PMS $(\mathrm{P}<0.01)$ and GS $(\mathrm{P}<0.05)$ (Fig 5e). Compared of NW, a decrease of POD activity was measured in GS floret before later mononuclear stage. Then POD activity increased after later mononuclear stage and reached top point in the stage of trinuclear (Fig 5f). CAT activities slowly increased in florets of all plants before late mononuclear stage, but sharply increased after late mononuclear stage. CAT activities of GS floret was highest in the stage of binuclear, and maintained this level to the trinuclear stage, while that of PMS was raising slower than NW after the binuclear stage(Fig $5 \mathrm{~g}$ ). MDA content of 
floret was higher than NW in the PMS in the process of the whole development, and it was significant higher $(\mathrm{P}<0.01)$ in the stage of meiosis. In the GS floret, MDA content was significant higher $(\mathrm{P}<0.01)$ than $\mathrm{NW}$ in the stage of meiosis, tetrad and trinuclear. And the content of MDA presented a trend of high to low in the three plants (Fig 5h).
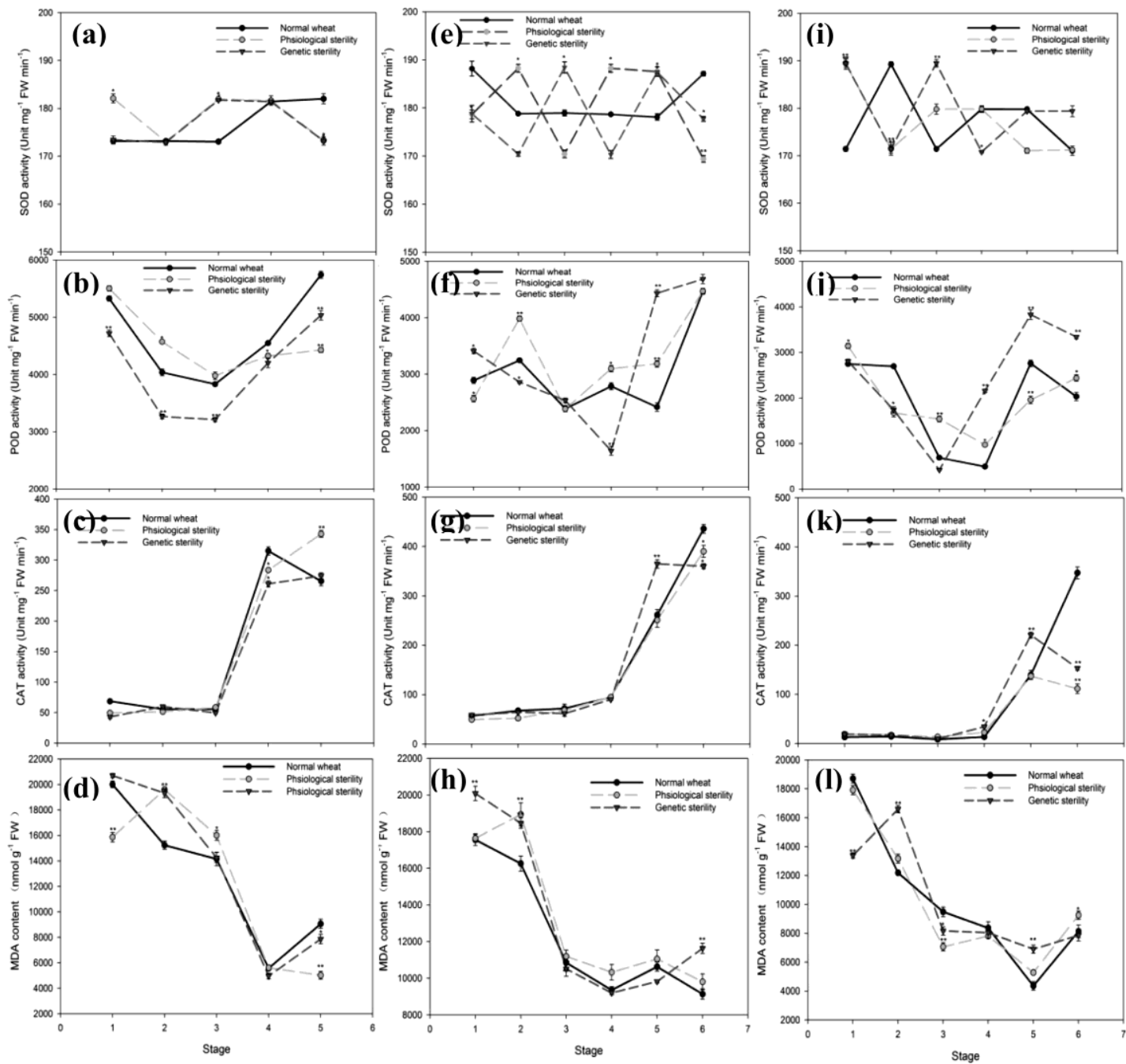

Fig 5. Activity of antioxidant enzymes and MDA content of spikes, florets and rachilla.

1 , meiophase stage; 2 , tetrad stage; 3 , early mononuclear stage; 4 , late mononuclear stage; 5 , binuclear stage; 6 , trinuclear stage. $n=3$, * Indicate $\mathrm{P}<0.05$ significant difference; ** Indicate $\mathrm{P}<0.01$ significant difference. (collete materials according to anther development period).

The level of SOD activities was opposite trend between the NW rachilla and the rachilla of PMS and GS (Fig 5i). The level of POD activities was a trend of "high-low-high", but compared with NW rachilla, PMS and GS had a larger diviation (Fig 5j). For CAT activities, it had the same trend before the binuclear stage in the NW and PMS and GS, while it was significant lower $(\mathrm{P}<0.01)$ than $\mathrm{NW}$ in the PMS and GS in the stage of trinuclear stage (Fig 5k). MDA concent of PMS and GS was higher than NW in the stage of meiosis and binuclear. When the anther development to the stage of trinuclear, MDA content of PMS rachilla was significantly higher $(\mathrm{P}<0.05)$ than that of NW (Fig 5l).

For SOD activity in the ovary, there were significantly $(p<0.01)$ decrease among the anther development to the stage of meiosis to mononuclear. And SOD activity remained the same level in the whole development process of GS ovary. POD activity, CAT activity and MDA content had similar variation tendency in the ovary of NW, PMS and GS. MDA content of ovary had almost no different in the anther stage of trinuclear in the NW, PMS and GS (Fig 6 a to $6 d)$.

From Fig 6e, we found the SOD activity at the meiosis of anther was significantly higher $(\mathrm{P}<0.01)$ than $\mathrm{NW}$ in the PMS and GS. Then this effect was no more pronounced along with the anther development to the stage of mononuclear. And then SOD activity of NW anther was ascend from the mononuclear stage to the binuclear stage, but no happened in the PMS and GS anther. There were significantly different $(\mathrm{P}<0.01)$ at the activity of SOD in the binuclear anther of PMS and GS than NW. From the 
binuclear stage to the trinuclear stage of anther, SOD activity of NW and PMS showed a trend of decline, while, the PMS was more lower $(\mathrm{P}<0.01)$ than $\mathrm{NW}$ in the trinuclear stage, and at this period, SOD activity of GS was in a stable state.
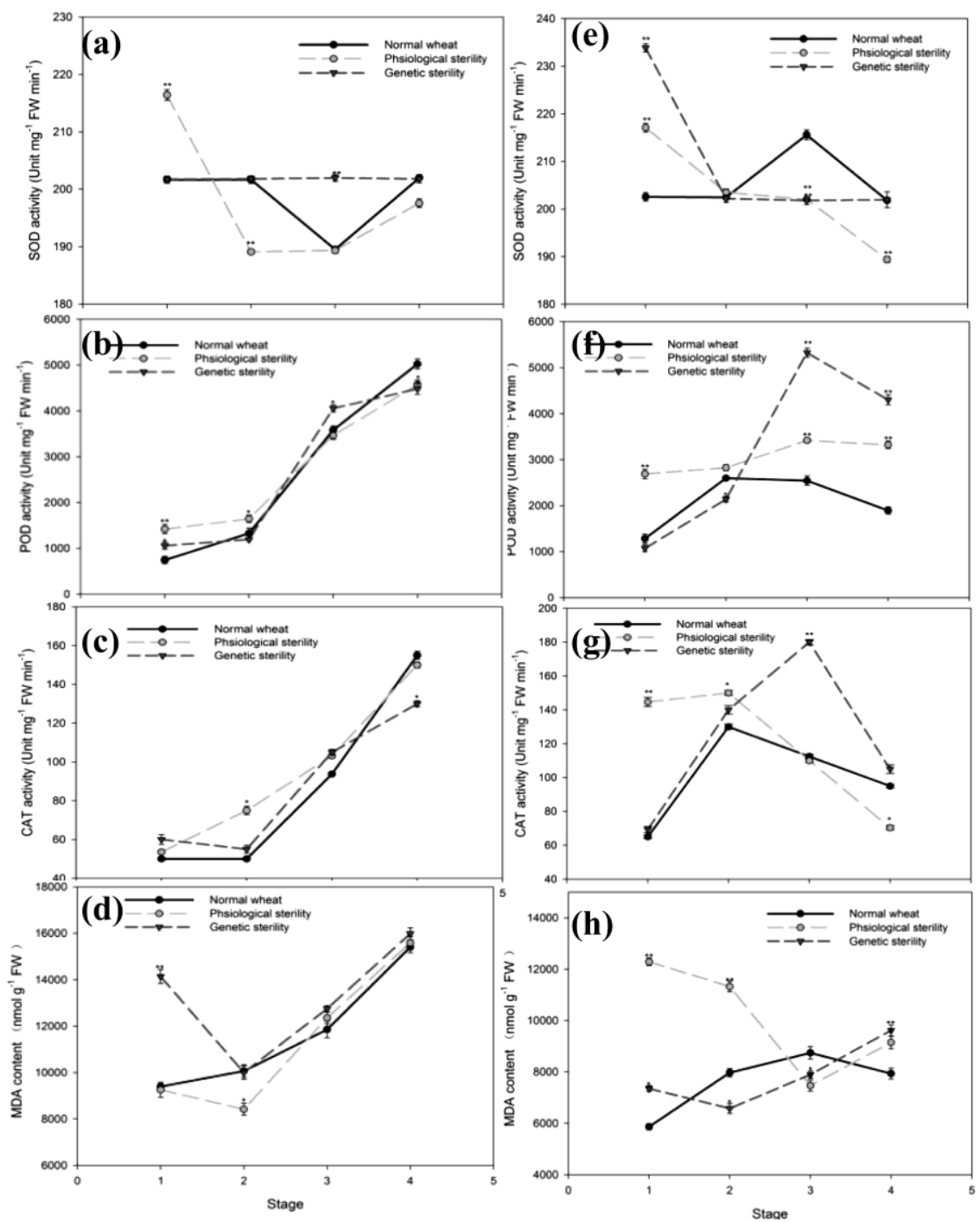

Fig 6. Activity of antioxidant enzymes and MDA content of ovary and anther.

1, tetrad stage; 2 , mononuclear stage; 3 , binuclear stage; 4 , trinuclear stage. $\mathrm{n}=3$, * Indicate $\mathrm{P}<0.05$ significant difference; ** Indicate $\mathrm{P}<0.01$ significant difference.

At the meiosis stage of anther, POD activity and CAT activity were significantly higher $(\mathrm{P}<0.01)$ than $\mathrm{NW}$ in the PMS plant, compared with NW, which showed a percentage increase of $209.28 \%$ and $222.53 \%$. CAT activity of the mononuclear anther was $15 \%$ higher than NW in PMS, and then it was linear decrease until to the trinuclear stage in the NW and PMS plants. CAT activity of GS anther was $63 \%$ higher than that of NW in the binuclear stage, and it was higher than NW at all times. And CAT activity of the trinuclear stage of PMS was significantly lower than that of NW. Whereas POD activity of the binuclear and the trinuclear stage anthers of PMS and GS were more significantly higher $(\mathrm{P}<0.01)$ than that of NW (Figs 6f and 6g). The MDA content of the meiosis stage anthers in PMS was $209.46 \%$ higher than that of NW.
Development to the stage of mononuclear, MDA content of PMS anther was $142.13 \%$ higher than the content of NW anthers. Interestingly, MDA content of PMS anther and NW anther appeared opposite trend in the process of anther development. In the stage of binuclear, PMS anther's MDA content fell to the lowest level, while, that of NW rose to the highest level. In GS anther, MDA content also appeared opposite trend against NW anther in the period of meiosis to mononuclear and the stage of binuclear to trinuclear. In the stage of trinuclear, MDA content of PMS anther and GS anther were significantly higher than NW anther (Fig 6h). MDA content and scavenging-enzyme activities had difference in the sterile and fertile leaves, spikes, florets, rachillaes at different anther stages, indicating that different 
tissues of ROS metabolism had noticeable difference in male sterility and normal wheat.

\section{DISCUSSION}

Membrane lipid stability is the basic condition to maintain normal physiological activities. MDA concentration is usually used to measure the extent of lipid peroxidation caused by oxidative stress. To some extent, the accumulation of MDA could be reflected the accumulation state of free radicals and the degree of membrane peroxidation (Wang et al., 2008). We demonstrate that the MDA concentration of wheat leaves had different in PMS and GS which compared with NW plants. The PMS plants treated with CHA-SQ-1 had higher MDA concentration than NW plants after the anther stage of tetrad in the top second leaf and flag leaf, which suggested that may be affected the normal photosynthesis of chloroplasts and respiration of mitochondrial. Antioxidant enzymes of SOD and CAT were mainly lower than NW in PMS and GS in the top second leaf and flag leaf, but the activity of POD in PMS leaf was higher than NW. Metabolic disorders of antioxidant enzymes in the top second leaf and flag leaf may have effects on plant life activities.

Previous studies provided evidence that well-equipped antioxidant defence plays an important role in minimizing water stress-induced spikelet sterility in upland rice (Selote and Khanna-Chopra, 2004). It was assumed that plant undergo significant metabolic changes in response to the membrane lipid peroxidation from the results of MDA content in the PMS and GS spikes and florets. The reason of the MDA content of PMS spikes and florets higher than that of NW may be induced by CHA-SQ-1, while the GS results may be the impact of its sterility gene. It was nearly the same of the changing trend of the activities of SOD, POD and CAT, but had different between male sterility lines and normal wheat. In the anther stage of trinuclear, antioxidant enzymes had significant different between male sterility lines and fertility wheat. These different of antioxidant enzymes and MDA content of trinuclear spikes and florets were consistent with the phenomena of the trinuclear stage anther and pollen grains (Fig 2).

Rachilla is one of the most important part that connected to the vegetative organs and reproductive organs. The transportation way and the mode of discharge of nutrient was affected the accumulation of photosynthate in the library origins directly (Smith et al., 1995). In this research, we found that antioxidant enzymes activities of rachilla had different between male sterility lines and fertility wheat. Rachilla MDA contents of PMS and GS were higher than NW in the anther stage of treted and binuclear, which showed MDA accumulation of rachilla may also affected the transportation of nutrients and material. While the antioxidant enzymes of rachilla also great changes have taken place, especially SOD and CAT activities in the anther stage of trinuclear (Fig 5).

The ovary of PMS and GS can normal pollination and bearing, and antioxidant enzymes and MDA content of ovary have a roughly similar trends. The content of MDA of ovary was almost no great difference after the anther stage of mononuclear in the PMS and GS and NW. This indicated that the ovary of PMS and GS had little toxic action by MDA in the development (Fig 6d).

Compared with ovary, there were significant differences in the activities of antioxidant enzymes and MDA content of anther. The result of MDA content of anther indicated that membranous peroxide was serious in the stage of tetrad and mononuclear in PMS plants. The period of tetrad to mononuclear was critical period of anther abortive. The result is in accordance with the result of Warmke et al. and Wan et al. (Wan et al., 2007; Warmke and Lee, 1978). In GS plants, sterility genes might play a key role, and MDA content reached highest in the trinuclear stage. These findings were in good agreement with the KI$\mathrm{I}_{2}$ staining result and SEM results, and membrane lipid peroxidation might be one reason of defective in the anther wall and pollen grains of PMS and GS plants in the development process (Fig 2). SOD and CAT activities were significant decrease along with the anther development. In the stage of tetrad, SOD and CAT had high activities could be the result of a substantial accumulation of ROS which induce the activities of antioxidant enzymes rise. The multiplicity role of POD was not only certain protective effect but also it was involved in the degradation of chlorophyll, and it was the product of plants at a certain stage of aging. In addition, the POD activity played a key role on the control the level of IAA change in plant. IAA content is closely related to the accumulation of nutrients in anther. Higher POD activity in sterile lines can lead to the IAA greatly decomposition and oxidation, which causing IAA deficiency in the process of male organ development and causing a further substance metabolism disorder, energy loss, leading to the occurrence of male sterility(Banga et al., 1984; Tripathi et al., 1983). In this study, the POD activity was always higher than NW in PMS, which was consistent with the above research results of IAA deficiency leading to the occurrence of male sterility. In conclusion, the disorder of scavenging-enzyme activities and accumulation of MDA and ROS in the male organ and supplier of nutrition blades was causing a further substance metabolism disorder, energy loss, leading to the occurrence of male sterility.

\section{ACKNOWLEDGMENTS}

This work was supported by National High Technology Research and Development Program of China (Nos.2011AA10A106), National Support Program of China (Nos: 2015BAD27B01), National Natural Science Foundation of China (Nos.31071477,31171611), Technological Innovation and Over Planning Projects of Shaanxi province (No.2014KTZB02-01-02) and The Key Scientific and Technological Innovation Special Projects of Shaanxi province "13115" (No.2010ZDKG-68, 2011KTZB02-01-01) and The Key Scientific Research Project in Colleges and Universities of Henan Province(No.15A210015). 


\section{LITERATURE CITED}

Ba, Q.S., G.S. Zhang, J.S. Wang, H.X. Che, H.Z. Liu, N. Niu, S.C. Ma, J.W. Wang. 2013.Relationship between metabolism of reactive oxygen species and chemically induced male sterility in wheat (Triticum aestivum L.). Can J Plant Sci. 93:1-7.

Bailly, C. 2004. Active oxygen species and antioxidants in seed biology. Seed Sci Res.14:93-107.

Banga, S., K. Labana, S.K. Banga. 1984. Male sterility in Indian mustard (Brassica juncea (L.) Coss.) - a biochemical characterization. Theor Appl Genet. 67:515-519.

Bhaskaran, J. and R. Panneerselvam. 2013. Accelerated Reactive Oxygen Scavenging System and Membrane Integrity of Two Panicum Species Varying in Salt Tolerance. Cell Biochem Biophys.3:885-892.

Darkó, É., H. Ambrus, É. Stefanovits-Bányai, J. Fodor, F. Bakos, B. Barnabás. 2004. Aluminium toxicity, Al tolerance and oxidative stress in an Al-sensitive wheat genotype and in Altolerant lines developed by in vitro microspore selection. Plant Sci. 166:583-591.

Deng, M. H., J.F. Wen, J.L. Huo, H.S. Zhu, X.Z. Dai, Z.Q. Zhang, H. Zhou, X.X. Zou. 2012. Relationship of metabolism of reactive oxygen species with cytoplasmic male sterility in pepper $(<$ i $>$ Capsicum annuum $</ i>$ L.). Scientia Hortic.134:232-236.

Dou, X.Y., K.Z. Yang, Y. Zhang, W. Wang, X.L. Liu, L.Q. Chen, X.Q. Zhang, D. Ye. 2011.WBC27, an Adenosine Triphosphate-binding Cassette Protein, Controls Pollen Wall Formation and Patterning in Arabidopsis. J Integr Plant Biol. 53:74-88.

Draganic, I., S. Lekic, T. Brankovic, G. Todorovic. 2011. Fatty acids and tocopherol content in sunflower seeds affected by accelerated ageing and priming with antioxidant solutions. Turk J Field Crops. 16, 100-104.

Fridovich I. 1978. The biology of oxygen radicals. Science. 201:875-880.

Gill, S.S. and N. Tuteja. 2010. Reactive oxygen species and antioxidant machinery in abiotic stress tolerance in crop plants. Plant Physiol Bioch. 48:909-930.

Hodges, D.M., J.M. DeLong, C.F. Forney, R.K. Prange. 1999. Improving the thiobarbituric acid-reactive-substances assay for estimating lipid peroxidation in plant tissues containing anthocyanin and other interfering compounds. Planta. 207:604-611.

Jia Z., W.H. Lin, H.B. Luo, L.Z. Sun, C. Liu. 2009. Comparison the activities of peroxidase, catalase, superoxide dismutase and peroxidase zymogram in C-cytoplasmic male sterility line on maize. Journal of Maize Sciences 6:014.

Jiang, M. and J. Zhang. 2001. Effect of abscisic acid on active oxygen species, antioxidative defence system and oxidative damage in leaves of maize seedlings. Plant Cell Physiol. 42:1265-1273.

Jiang, P.D., X.Q. Zhang, Y.G. Zhu, W. Zhu, H.Y. Xie, X.D. Wang. 2007. Metabolism of reactive oxygen species in cotton cytoplasmic male sterility and its restoration. Plant Cell Rep. 26:1627-1634.

Li, S.Q., C.X. Wan, J. Kong, Z.J. Zhang, S.Y. Li, Y.G. Zhu. 2004. Programmed cell death during microgenesis in a Honglian
CMS line of rice is correlated with oxidative stress in mitochondria. Funct Plant Biol. 31:369-376.

Liu, Z.N., X.L. Yu, F.Z. Wang, S. Hu, Y.P. Liu, G. Lu. 2012. Physiological, biochemical, and molecular characterization of a new female sterile mutant in turnip. Plant Growth Regul. 68:239-248

Li, M.H., X.Y. Zhang, Y.F. Fan, Y.K. Li, Y.L. CAO. 2008. A study on activated oxygen metabolism in the male sterile plants of lycium barbarum. Acta Agriculturae Universitatis Jiangxiensis 5:009.

Mittler, R. 2002. Oxidative stress, antioxidants and stress tolerance. Trends Plant Sci 7:405-410.

Ou, L.J., Z.Q. Zhang, X.Z. Dai, X.X. Zou. 2013. Photooxidation Tolerance Characters of a New Purple Pepper. PloS One. 8:e63593.

Polidoros, A.N. and J.G. Scandalios. 1999. Role of hydrogen peroxide and different classes of antioxidants in the regulation of catalase and glutathione S-transferase gene expression in maize (Zea mays L.). Physiol Plantarum. 106:112-120.

Selote, D.S. and R. Khanna-Chopra 2004. Drought-induced spikelet sterility is associated with an inefficient antioxidant defence in rice panicles. Physiol Plantarum. 121:462-471.

Smith, A.M., K. Denyer, C.R. Martin. 1995. What controls the amount and structure of starch in storage organs? Plant Physiol. 107:673.

Sunkar, R., D. Bartels, H.H. Kirch. 2003. Overexpression of a stress-inducible aldehyde dehydrogenase gene from Arabidopsis thaliana in transgenic plants improves stress tolerance. Plant J. 35:452-464.

Tripathi, D., S. Mehta, N. Rao. 1983. Soluble proteins and isoenzymes from seeds of diverse male steriles of sorghum,(Sorghum bicolor (L.) Moench). Theor Appl Genet. 64:325-331.

Wan, C.X., S.Q. Li, L. Wen, J. Kong, K. Wang, Y.G. Zhu. 2007. Damage of oxidative stress on mitochondria during microspores development in Honglian CMS line of rice. Plant Cell Rep. 26:373-382.

Wang, J.S., G.S. Zhang, L. Yuan, M.Z. Zhang, N. Niu, S.C. Ma, J.X. Ye. 2009. Metabolism of reactive oxygen species of physiological male-sterile anther induced by chemical hybrid agent in wheat. Acta Botanica Boreali-Occidentalia Sinica 7:013.

Wang Z., Y.X. Zhang, Z.B. Huang, L. Huang. 2008. Antioxidative response of metal-accumulator and nonaccumulator plants under cadmium stress. Plant Soil. 310:137-149.

Warmke, H.E., S.L.J. Lee. 1978. Pollen abortion in T cytoplasmic male-sterile corn (Zea mays): a suggested mechanism. Science. 200:561-563.

Winterbourn, C.C., R. Hawkins, M. Brian, R. Carrell. 1975. The estimation of red cell superoxide dismutase activity. J Lab Clin Med. 85:337-341.

Fatma, A. T., Lker. Emre, T. Muzaffer. 2010. A study to incorporate high protein content from tetraploid wheat $(\mathrm{T}$. turgidum dicoccoides) wheat (T. aestivum vulgare). Turk J Field Crops. 15(1): 69-72 